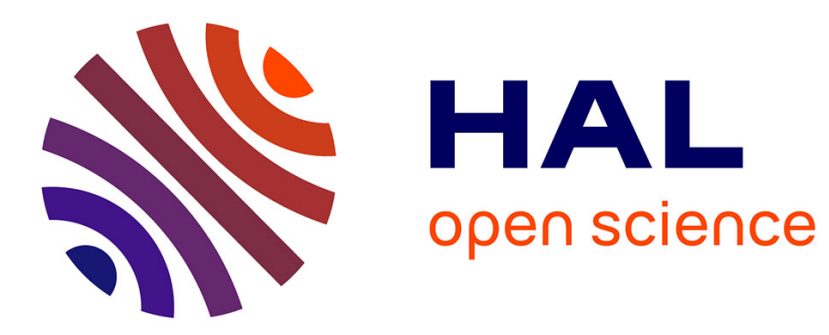

\title{
Effect of high current density on the admittance response of interface states in ultrathin MIS tunnel junctions.
}

Christian Godet, Alain-Bruno Fadjie-Djomkam, Soraya Ababou-Girard

\section{- To cite this version:}

Christian Godet, Alain-Bruno Fadjie-Djomkam, Soraya Ababou-Girard. Effect of high current density on the admittance response of interface states in ultrathin MIS tunnel junctions.. Solid-State Electronics, 2013, 80, pp.142-151. 10.1016/J.SSE.2012.10.012 . hal-00815698

\section{HAL Id: hal-00815698 https://hal.science/hal-00815698}

Submitted on 31 Mar 2014

HAL is a multi-disciplinary open access archive for the deposit and dissemination of scientific research documents, whether they are published or not. The documents may come from teaching and research institutions in France or abroad, or from public or private research centers.
L'archive ouverte pluridisciplinaire HAL, est destinée au dépôt et à la diffusion de documents scientifiques de niveau recherche, publiés ou non, émanant des établissements d'enseignement et de recherche français ou étrangers, des laboratoires publics ou privés. 


\section{Effect of high current density on the admittance response}

\section{of interface states in ultrathin MIS tunnel junctions}

Christian Godet, ${ }^{a}$ Alain-Bruno Fadjie-Djomkam, Soraya Ababou-Girard, Institut de Physique de Rennes, CNRS UMR 6251, Université de Rennes 1, Campus de Beaulieu, 35042 Rennes Cedex, France

a) Author to whom correspondence should be addressed ;

electronic mail: christian.godet@univ-rennes1.fr

\section{Corresponding author:}

Christian GODET

EPSI - IPR (Bât. 11C - Beaulieu)

Université Rennes 1

35042 RENNES (France)

Tél : +33223235706

Fax: +33223236198

Mél : christian.godet@univ-rennes1.fr 


\section{Abstract}

The effect of a high current density on the measured admittance of ultrathin Metal - Insulator Semiconductor (MIS) tunnel junctions is investigated to obtain a reliable energy distribution of the density, $D_{S}(E)$, of defects localized at the semiconductor interface. The behavior of admittance $Y(V, T, \omega)$ and current density $J(V, T)$ characteristics is illustrated by rectifying $\mathrm{Hg} / /$ $\mathrm{C}_{12} \mathrm{H}_{25}$ - Si junctions incorporating $n$-alkyl molecular layers (1.45 $\mathrm{nm}$ thick) covalently bonded to n-type Si(111). Modeling the forward bias admittance of a non equilibrium tunnel junction reveals several regimes which can be observed either in $C(\omega \approx 0)$ vs $(J)$ plots of the low frequency capacitance over six decades in current or in $M^{m}(\omega)$ plots of the electrical modulus over eight decades in frequency. At low current density, the response of interface states above mid-gap is unaffected and a good agreement is found between the interface states densities derived from the modeling of device response time $\tau_{\mathrm{R}}(V)$ and from the low-high frequency capacitance method valid for thick MIS devices; the low defect density near mid-gap $\left(D_{S}<10^{11}\right.$ $\mathrm{eV}^{-1} . \mathrm{cm}^{-2}$ ) results from a good passivation of dangling bonds at the $\mathrm{C}_{12} \mathrm{H}_{25}-n$ Si interface. In the high current density regime $\left(/>1 \mathrm{~mA} . \mathrm{cm}^{-2}\right)$, the admittance depends strongly on both the density of localized states and the dc current density, so that the excess capacitance method overestimates $D_{s}$. For very high current densities $\left(J>10 \mathrm{~mA} \cdot \mathrm{cm}^{-2}\right)$, the observation of a linear $C(\omega \approx 0)$ vs $(J)$ dependence could indicate some Fermi level pinning in a high interface density of states located near the Si conduction band. The temperature-independent excess capacitance $C(\omega \approx 0)-C(1$ $\mathrm{MHz}$ ) observed at very small $J$, not predicted by the admittance model, is attributed to some dipolar relaxation in the molecular junction. 


\section{Introduction}

The development of Metal-Insulator-Semiconductor (MIS) junctions with ultra-thin insulator $\left(d_{\mathrm{T}}<3 \mathrm{~nm}\right.$ ) has stimulated the study of new insulating materials, such as saturated organic chains with a wide electronic band gap $(\approx 7 \mathrm{eV}) .{ }^{1}$ In the field of hybrid molecular-silicon junctions, alkyl-based devices have been investigated for fundamental studies of transport mechanisms (thermionic emission, tunnelling, recombination) while applications are foreseen in the fields of solar cells and gate insulators. ${ }^{2,3}$ Alkyl monolayers may form a well controlled interlayer for further assembly of nanoscale objects (molecules, nanoparticles, nanotubes) providing new functionalities to form original devices and circuit architectures. ${ }^{4-8}$

Covalent binding of linear saturated ( $n$-alkyl) chains to hydrogenated $\mathrm{Si}(111): \mathrm{H}$ surfaces ${ }^{9}$ forms robust molecular layers with a high coverage which play the role of a nanometer-thick tunnel barrier. Due to sterical constraints, the density of surface Si-H sites which are not grafted with alkyl molecules is larger than $4 \times 10^{14} \mathrm{~cm}^{-2}$ ( $\approx 60 \%$ of surface Si atom density); however, the density of electrically active defects, $D_{S}$, remains relatively small, at least immediately after grafting, in the $1 \times 10^{10}-3 \times 10^{11} \mathrm{eV}^{-1} \cdot \mathrm{cm}^{-2}$ range. ${ }^{10-15}$ However, besides the problem of engineering a homogeneous and reliable top electrode in contact with a nanometer-thick molecular monolayer, a major issue in hybrid molecular-silicon junctions is the structural disorder which results from the irreversible grafting mechanism related to the strong covalent bonding ; hence structural disorder probably induces some distribution of barrier heights for electrical transport and may explain the wide range of conductance values observed experimentally. ${ }^{16,17}$

In metal / semiconductor (SC) junctions, the presence of interface states and of a thin insulating layer have been recognized as non idealizing factors in the current-voltage $(I-V)$ characteristics. $^{18-21}$ A number of methods based on non-stationary measurements have been developed for interface defect characterization in thick MIS diodes ${ }^{22,23}$ and tentatively extended to MIS junctions with interfacial layers, ${ }^{24,25}$ ultrathin $\mathrm{SiO}_{2},{ }^{19,26-29}$ and molecular insulator. ${ }^{3,14,15,30}$ 
In the latter devices, frequency and temperature dependence of molecular MIS admittance brings valuable information to discriminate interface states response and dipolar relaxation effects. ${ }^{14,31-33}$

MIS tunnel diodes are non equilibrium devices due to the large current density flowing through the thin insulating layer (Figure 1). It has been shown that, between 1 and $2 \mathrm{~nm}$ thick $\mathrm{SiO}_{2}$, the quasistatic hypothesis progressively fails and a kinetic approach should be developed; ${ }^{34}$ this effect is expected to occur at larger thickness values for an insulator with a lower tunnel barrier height. We must then calculate the position of the quasi-Fermi level at the interface, its value depending on the applied voltage, on the transmission coefficient of the barrier (i.e. essentially on the insulator thickness) and on the interface defects. The non-stationary transport properties (capacitance and conductance) of ultrathin MIS tunnel junctions and non-abrupt MS junctions under modulated bias, $V+V_{A C} \exp (j \omega t)$, have been derived by Gomila ${ }^{35,36}$ using coupled kinetic equations which define the occupancy of interface states as a function of applied dc bias, $V$, bias modulation frequency, $\omega$, and temperature, $T$. In contrast with previous models, exchange of carriers between interface states and either the metal or the semiconductor has been considered. However, to our best knowledge, no systematic validation of Gomila's model has been performed with experimental data for tunnel MIS diodes.

In this work, the behavior of admittance $Y(V, T, \omega)$ and dc current density $J(V, T)$ characteristics in ultrathin MIS tunnel junctions is illustrated by $\mathrm{Hg} / / \mathrm{C}_{12} \mathrm{H}_{25}-n$ Si junctions incorporating $n$-alkyl molecular layers $\left(d_{T}=1.45 \mathrm{~nm}\right)$ covalently bonded to $n$-type $\operatorname{Si}(111)$. In order to obtain a reliable density of states distribution from admittance measurements in non equilibrium MIS devices, this work emphasizes the combined role of dc current density and interface states density, as predicted by the tunnel barrier model. The strong rectification in $J(V$, $T$ ) due to the $n$-type doping provides a very large range of dc current which is helpful to identify several transport regimes. 
The organization of the manuscript is as follows. Section 2 recalls the physics of the tunnel barrier model leading to the admittance $Y(V, T, \omega)$ of ultrathin MIS tunnel junctions. The combined role of dc current density $J(V, T)$ and interface states density distribution $D_{S}(E)$ in the measured admittance characteristics, in particular the low frequency capacitance and high frequency conductance, is emphasized and the device response time is introduced. Section 3 describes the experimental methods, including photochemical grafting of alkene molecules on Si(111), X-ray photoelectron spectroscopy (XPS) characterization of molecular coverage, spectroscopic ellipsometry (SE), dc and ac electrical transport at variable $T$. Besides $J(V, T)$ measurements in the $T$ range $243-293 \mathrm{~K}$, the admittance $Y(V, T, \omega)$ is characterized in a wide frequency range $\left(10^{-2}\right.$ to $\left.10^{7} \mathrm{~Hz}\right)$ in order to investigate the dynamic characteristics of the tunnel barrier, the space charge region and the interface trap distribution. Section $\mathbf{4}$ is devoted to the admittance spectroscopy characteristics, used to obtain the flat band voltage, $V_{\mathrm{FB}}$, the device response time, $\tau_{\mathrm{R}}$, and the density of interface electronic states, $D_{S}(E)$. The comparison of the density of states distributions derived from the device response time and from the classical low high frequency capacitance method (valid for thick MIS devices), respectively, allows to define a "low current density regime" and a "high current density regime". The potential influence of high local current density, e.g. due to some lateral inhomogeneity of the barrier height, on the measured admittance $Y(V, T, \omega)$ is also discussed.

Our experimental results are compared with some predictions of admittance modeling for ultrathin MIS tunnel junctions, in particular the dependence of the low frequency capacitance $C(\omega \approx 0)$ on $J(V, T)$ and the frequency dependence of the electrical modulus. Some model parameters such as the microscopic response time of interface defects and their distribution in energy, $D_{S}(E)$, are derived from experimental data. While all of the experimental results presented in this work were obtained using $\mathrm{Hg} / / \mathrm{C}_{12} \mathrm{H}_{25}-n \mathrm{Si}$ (111) molecular junctions, the method is more general and could be applied to any tunnel MIS device. 


\section{Non stationary transport model}

The non-stationary transport properties (capacitance and conductance) of non intimate Schottky contacts and MIS tunnel diodes have been derived using coupled kinetic equations which define the occupancy of interface states as a function of applied dc bias, bias modulation frequency and temperature. ${ }^{35,36}$ We briefly recall the main hypothesis and the device admittance expression to emphasize the combined role of dc current density $J(V, T)$ and interface states density distribution $D_{S}\left(E_{C}-E_{T}\right)$ in the low frequency capacitance and high frequency conductance and to introduce the device response time directly evidenced in $M^{\prime \prime}(\omega)$ plots of the electrical modulus.

\subsection{Kinetic mechanisms}

In the energy band diagram of the MIS tunnel diode with insulator thickness $d_{T}$ (noted as $\delta$ in Figure 1), the barrier height, $\phi_{B N}$, is defined by the metal work function, $\phi_{m p}$ and the SC electron affinity, $\chi$. The dc thermionic emission current across the interface is governed by this barrier height and by an attenuation factor $\exp \left(-\beta^{\circ} d_{T}\right)$ due to the tunnel barrier transparency; the parameter $\beta^{0}$ can be derived from the temperature dependence of the apparent barrier height in the thermionic emission regime. ${ }^{15}$

Figure 1 shows that three elementary kinetic processes can be identified:

$$
q_{\mathrm{n}} \leftrightarrow q_{\mathrm{m}} ; \quad q_{\mathrm{s}} \leftrightarrow q_{\mathrm{m}} ; q_{\mathrm{s}} \leftrightarrow q_{\mathrm{n}}
$$

in which $q_{\mathrm{n}}, q_{\mathrm{m}}, q_{\mathrm{s}}$ stand respectively for the electrons in the semiconductor, in the metal and at the interface states. If the series resistance $\left(R_{S}\right)$ can be neglected (not true at high $I$ ), the applied bias $V$ is the difference $F_{m}-F_{n}$ between the metal Fermi level, $F_{m}$, and the semiconductor Fermi level, $F_{n}$. 
In contrast with Gomila's approach, where no restriction on the carrier exchange between interface states and either the metal or the semiconductor has been assumed, previous models of thin MIS junctions restricted the exchange of electrical carriers of interface traps to the SC conduction band. ${ }^{25,29,37,38}$ Although resulting expressions of the admittance are quite similar to Equation 12, the microscopic response times of localized interface states are quite different: a capture-emission time constant is derived from Shockley-Read-Hall parameters ${ }^{22}$ in the former models, ${ }^{37-40}$ in contrast with the tunnel response time (Eqn 7) in Gomila's model. ${ }^{36}$

\subsection{Device admittance}

The influence of the dc current density on the device admittance is qualitatively described by the "trap transistor model": the out-of-phase modulation of interface charge $\left(Q_{S S}\right)$ is responsible for an out-of-phase modulation of the electric field in the insulator and of the SC band bending $\delta \Psi_{\mathrm{S}}$, which in turn produces an ac-current $\delta J=J\left(q \delta \psi_{S} / k T\right)$ and results in a large capacitance at low frequency $C(\omega \approx 0){ }^{25}$

The voltage drop $\Delta$ on the insulator (Figure 1) is related to the accumulated charge per unit area at the SC surface and in the space-charge layer (Gauss's law):

$$
\Delta=\left(\phi_{m}-\chi\right)-\phi_{B N}=-\frac{q}{C_{i}}\left(n_{S S}\left(F_{S}-E_{V}\right)-N_{D S}\right)+\frac{Q_{S C}}{C_{i}}
$$

where the number $n_{S S}\left(F_{S}-E_{W}\right)$ of occupied interface states per unit area is a function of the surface Fermi level position $\left(F_{S}\right)$ with respect to the valence band energy $\left(E_{W}\right), N_{D S}$ is the density of donor states at the interface. The charge in the semiconductor, $Q_{S C}=\left(2 q \varepsilon_{S} N_{D} \psi_{S}\right)^{1 / 2}$, is given by the permittivity $\varepsilon_{S y}$ the dopant density $N_{D y}$ and the band bending $\psi_{S} \cdot C_{S C}=\varepsilon_{S} / w$ and $C_{\tilde{\tilde{I}}}$ are respectively the SC and the insulator capacitance values per unit area.

All relevant transport parameters can be determined from the position of the surface Fermi level, $F_{s}$, and the transition coefficients $\left(\lambda_{n m,} \lambda_{a m}, \lambda_{s n}\right)$ corresponding to the elementary 
processes represented in Equation 1. Note that coefficients $\lambda_{m m}$ and $\lambda_{\operatorname{sm}}\left(\mathrm{cm}^{-2} \mathrm{~s}^{-1}\right)$ both incorporate a tunnel barrier attenuation coefficient, $\exp \left(-\beta^{\circ} d_{T}\right)$. The total current density $J$ flowing through the system is given by $l=\frac{\partial D}{\partial t}+J_{n}$, where $D=\varepsilon E$ and $J_{n}=J_{n}^{T E}+\frac{J_{S}}{1+\alpha}$ is the sum of thermionic emission and interface states current densities:

$$
\begin{aligned}
& I_{n}^{T E}=-q N_{C} V_{R} \exp \left(-q \beta \phi_{b n}\right)\left[1-\exp \left(\beta\left(F_{n}-F_{m}\right)\right)\right] \\
& I_{s}=-q \lambda_{n s}(1+\alpha) \exp \left(-q \beta \phi_{b n}\right)\left(\exp \left(\beta\left(F_{g}-F_{m}\right)\right)-\frac{1+\alpha^{-1} \exp \left(\beta\left(F_{n}-F_{m}\right)\right)}{1+\alpha^{-1}}\right) \\
& \alpha=\frac{\lambda_{g m}}{\lambda_{g m}}=\exp \left(\frac{q V_{C}}{k T}\right) \text { and } N_{C} V_{R}=\lambda_{n m}+\frac{\exp \left(E_{G} / k T\right)}{\lambda_{g m}^{-1}+\lambda_{g m}}
\end{aligned}
$$

Here $\beta=1 / k T$ and $V_{C}$ is a critical bias (assumed to be a constant) which characterizes the interface states occupation under non equilibrium. ${ }^{35}$ Using the ac expression of the charge conservation equation $I_{s}+q \frac{\partial n_{S S}}{\partial t}=0$, at the interface states, one obtains the out-of-phase modulation of the surface Fermi level position

$\frac{\delta\left(F_{S}-F_{m}\right)}{\delta V}=q\left[\frac{\exp q \beta\left(V-V_{C}\right)}{(1+j \omega \tau)\left(1+\exp q \beta\left(V-V_{C}\right)\right)}\right]$

where

$\tau=\frac{D_{s a}}{\beta \lambda_{n s} \alpha \exp \left(-q \beta \phi \frac{d \sigma}{d \sigma}\right)}$

is the microscopic tunnel response time and

$D_{s a}=D_{s}\left(1-\frac{1}{q} \frac{\partial\left(F_{s}-F_{m}\right)}{\partial V}\right), \quad D_{s b}=D_{S}\left(\frac{1}{q} \frac{\partial\left(F_{s}-F_{m}\right)}{\partial V}\right), \quad D_{S}=\left.\frac{\partial n_{S S}}{\partial E_{F_{s}}}\right|_{E_{F S}-E_{V}^{Q}}$

are the fractions of the total density of states $D_{S}(E)$ in equilibrium with the metal or the SC, respectively. In ac mode, a small-signal analysis provides the total ac current

$$
\delta J=j \omega \delta Q_{S C}+\delta J_{n}^{T E}+\frac{\delta J_{S}}{1+\alpha}
$$


The expressions of the ac current and admittance simplify for forward bias values, $V>V_{C}$, where the fraction of interface states in equilibrium with the metal can be neglected $\left(D_{\widetilde{\sigma a}} \approx 0\right)$ hence $D_{S} \approx D_{a b}$, the density of states in equilibrium with the semiconductor. ${ }^{36}$ The junction admittance, $Y(\omega)=\delta J / \delta V_{s}$ is given by:

$Y(\omega)=G+j \omega C=q \beta J_{0}+\left[q \beta J_{d e}+\frac{G_{S}}{1+\alpha}+j \omega\left(C_{s c}+\frac{C_{S}}{1+\alpha}\right)\right] \times \frac{j \omega C_{\bar{i}}}{G_{S}+j \omega\left(C_{\bar{i}}+C_{S C}+C_{S}\right)}$

In Eqn. 10, the square bracket is $\left(\delta J / \delta \psi_{s}\right)$, the last fraction is $\left(\delta \psi_{s} / \delta \mathrm{V}\right)$ and the interface states capacitance and conductance per unit area, defined by the relation $q \delta J_{S}=\left(G_{S}+j \omega C_{S}\right) \delta \psi$, have been introduced:

$G_{S}=\omega^{2} \tau \frac{q^{2} D_{S}}{1+\omega^{2} \tau^{2}} \quad(11 a) \quad C_{S}=\frac{q^{2} D_{S}}{1+\omega^{2} \tau^{2}}$

Using $C_{\mathbb{E}}=C_{\mathrm{i}}+C_{\mathrm{SC}}$, the MIS conductance and capacitance per unit area are:

$$
\begin{aligned}
& \hat{G}=q \beta J_{0}+\frac{q \beta J C_{i}\left(C_{S}+C_{\Sigma}\right)+C_{i}^{2} G_{S}\left(\frac{1-\alpha C_{S C} / C_{\tilde{i}}}{1+\alpha}\right)}{\left(\frac{G_{S}}{\omega}\right)^{2}+\left(C_{S}+C_{\Sigma}\right)^{2}} \\
& \hat{C}=C_{\tilde{i}} \frac{q \beta J \frac{G_{S}}{\omega^{2}}+\frac{\left(G_{S} / \omega\right)^{2}}{1+\alpha}+\left(C_{S}+C_{\Sigma}\right)\left(\frac{C_{S}}{1+\alpha}+C_{S C}\right)}{\left(\frac{G_{S}}{\omega}\right)^{2}+\left(C_{S}+C_{\Sigma}\right)^{2}}
\end{aligned}
$$

We further derive approximations of the low frequency capacitance $C(0)$ and high frequency conductance $G(\infty)$

$\widehat{G}(\infty)=q \beta J_{0}+\frac{q \beta J C_{i} C_{\Sigma}+q^{2} D_{S} \tau^{-1}\left(\frac{1-\alpha C_{S C} / C_{\tilde{i}}}{1+\alpha}\right) C_{i}^{2}}{C_{\Sigma}^{2}}$ 
$\hat{C}(0)=C_{i} \frac{q \beta J q^{2} D_{s} \tau+\left(\frac{q^{2} D_{s}}{1+\alpha}+C_{s c}\right) C_{\bar{\Sigma}}}{C_{\bar{\Sigma}}{ }^{2}}$

which are particularly interesting to assess the role of the current density through the tunnel MIS diode in the measured admittance. Considering the role of $D_{S}, \tau$ and $J$ in admittance spectroscopy data, the $D_{S} \tau$ product and $J_{d e}$ are strongly coupled in the excess capacitance

$$
C(0)-C(\infty) \approx q^{2} D_{S}\left(1+\frac{q \beta J \pi}{C_{i}}\right)
$$

while only $D_{S} \tau^{-1}$ appears in the excess conductance due to interface states (for $q^{2} D_{S} \ll C_{i}$ )

$$
G(\infty)-G(0) \approx q^{2} D_{S} \tau^{-1}\left(C_{i} / C_{\Sigma}\right)^{2} \approx q^{2} D_{S} \tau^{-1}
$$

In principle, the magnitude of $\tau$ and $D_{s} \tau$ values can be derived from $C(0)$ vs $J$ plots in some particular conditions, since Eqn 15 predicts an inflexion point at $J \approx C_{i} / q \beta \tau$, whereas a linear regime is expected at very high current densities, if $D_{S}$ is a constant, with a slope $\left(q^{3} D_{S} \beta \tau / C_{i}\right)$.

Simulations of the admittance (Fig. 2-3) were performed using experimental current density $J(V)$ data (Figure 4), device area $S=5 \times 10^{-3} \mathrm{~cm}^{2}$ and variable $D_{S}$ values in the range $10^{8}-10^{13}$ $\mathrm{eV}^{-1} \cdot \mathrm{cm}^{-2}$. The respective effects of the current density (forward bias) and defect density on the conductance, capacitance and electrical modulus are shown in Figure 2.

For comparison with experimental results (Fig. 5-8), we have chosen to include in the model the effect of a series resistance $\left(R_{S}=100 \Omega\right)$ on the admittance. ${ }^{38}$ Note that high values of the series resistance $R_{S}$ limit the frequency range where interesting physical mechanisms can be observed, typically below $1 \mathrm{MHz}$ in this study. Complementary experiments have shown that the $R_{S}$ value can be decreased by using a higher Si doping level. As discussed in Section 4.2, $R_{S}$ can be obtained experimentally in the high frequency regime where $G(\omega)$ is proportional to $\omega^{2}$.

\subsection{Device response time}


For a parallel $R-C$ circuit, the calculated device response time, $\tau_{R}(V, T)=(C / G)$, is a physical quantity which can be readily compared with experimental values of the inverse angular frequency, $\tau_{R}=\omega_{M A X}^{-1}$, at the maximum of the imaginary part of either the impedance $Z^{\prime \prime}(\omega)$ or electrical modulus $M^{m}(\omega)$ plots. The effects of a high current density can be qualitatively derived for a junction with a low defect density $\left(C_{S}<<C_{S C}\right)$; in the low frequency limit $(\omega \pi \ll 1)$ the tunnel barrier model (Eqn 12) gives a junction response time $\tau_{\mathrm{R}}(V, T)$ which depends on the density of localized states $D_{S}$ and on the dc current density $/$ through the junction:

$\tau_{R} \approx \frac{q \beta J q^{2} D_{S} \tau+C_{S C} C_{\tilde{i}}}{(q \beta J) C_{\tilde{i}}}$

where $\tau$ is defined by Eqn 7. Hence, the response time is larger than in the ideal case $\left(\tau_{R} \approx \frac{C_{S C}}{q \beta J}\right)$ obtained with no defects $\left(D_{S}=0\right)$.

In this Section, we focus on the behavior of $M^{u}(\omega)=C_{0} \omega\left[G\left(1+R_{S} G\right)+R_{S} \omega^{2} C^{2}\right] /\left(G^{2}+\omega^{2} C^{2}\right)$, where we have included the effect of a series resistance $\left(R_{S}\right)$ on the measured admittance and $C_{0}$ is an arbitrary normalization factor (100 $\mathrm{pF}$ ). Using the same parameters as in Figure 2c and a current density $l=1 \times 10^{-7}$ A.cm ${ }^{-2}$ (corresponding to $+0.1 \mathrm{~V}$ bias and $293 \mathrm{~K}$ ), Figure 3a shows that $M^{\mathrm{m}}(\omega)$ plots present two maxima corresponding respectively to the semiconductor (LF) and to the tunnel barrier (HF). For very low $D_{S}$ values, the low frequency response occurs at $f^{L F}=\left(G / 2 \pi C_{S C}\right)$ while the high frequency response at $f^{H F}=1 / 2 \pi \tau$ is related to the microscopic tunnel response time.

The electrical modulus $M^{\prime \prime}(\omega)$ has been simulated over a wide range of $D_{S}$ values $\left(10^{8}-10^{13}\right.$ $\mathrm{eV}^{-1} \cdot \mathrm{cm}^{-2}$ ). Figure $\mathbf{3 b}$ shows that the two peaks shift in opposite directions with increasing density of localized states, namely $f^{L F}$ decreases because $C(0)$ increases while $f^{H F}$ increases because $G(\infty)$ increases. We have also reported the peak intensities which also change in 
opposite directions; basically, with increasing $D_{S}, M_{M A X}^{L F}$ decreases because $C(0)$ increases while $M_{M A X}^{H F}$ increases because $G(\infty)$ increases. Figure $3 \mathbf{b}$ corresponds to a low current density $\left(1 \times 10^{-7}\right.$ A. $\mathrm{cm}^{-2}$ ); however, with increasing forward bias, $f^{L F}$ will increase exponentially and may eventually approach $f^{H F}$, as illustrated by experimental data in Figure 8a.

Since the low frequency response dominates $M^{m}(\omega)$ plots in device-quality MIS tunnel junctions (typically $D_{S}<1 \times 10^{11} \mathrm{eV}^{-1} \cdot \mathrm{cm}^{-2}$ ), the experimental evolution of both $f^{L F}$ and $M_{M A X}^{L F}$ values may give valuable information on the distribution $D_{S}(E)$. These methods are essentially sensitive in the range $2 \times 10^{9}-2 \times 10^{11} \mathrm{eV}^{-1} \cdot \mathrm{cm}^{-2}$ where $M_{M A I}^{L F}$ decreases over one decade (Fig. $3 \mathbf{b}$ ). In a previous report, ${ }^{15}$ the response time $\tau_{R}\left(V_{y} T_{y} D_{S}\right)$ of the tunnel MIS device has been used to derive $D_{S}\left(E_{C}-E_{T}\right)$. In this work, a more complete analysis is proposed to check the consistency of both methods.

\subsection{Barrier height distribution}

An important assumption of the model is that the density of interface states and the current density through the device are laterally homogeneous. In the case of inhomogeneous interface potential providing low barrier height patches, it has been shown that a small fraction of the device area may be responsible for a significant fraction of the dc current through Schottky barrier devices, in particular at low bias and low temperature. ${ }^{39-41}$ A similar effect has been observed in molecular tunnel junctions, after ageing at the ambient. ${ }^{42}$

In this case, for a given applied bias value, low barrier height patches with a high local dc current density may result in a distribution of $\tau$ values (tailing towards shorter times, Eqn 7). As a consequence, the interface states conductance $G(\omega)$ is a sum (weighted by the effective patch area $\left.S_{i}\right)$ of local conductance values $G_{i}(\omega)$ defined by their characteristic angular frequency $\tau_{\tilde{i}}^{-1}$ and high frequency plateau $q^{2} D_{s} \tau_{i}^{-1}$ value (Eqn 16). A tailing of the $\tau_{i}^{-1}$ distribution (i.e. Gaussian or exponential) towards higher frequencies is expected to produce a sub-linear 
dependence of the integrated $G(\omega)$ vs $\omega$, rather than a plateau. However, since the band bending is different from patch to patch, a patch-dependent $D_{s}$ (determined by the position of the Fermi level at the interface) may also influence the shape of $G(\omega)$.

\section{Experimental}

Experimental methods including photochemical grafting, X-ray photoelectron spectroscopy (XPS), spectroscopic ellipsometry (SE), dc and ac electrical transport, were described in detail previously. ${ }^{15}$ Covalent grafting of 1-dodecene $\left(\mathrm{C}_{11} \mathrm{H}_{22}=\mathrm{CH}_{2}\right)$ molecules (puriss, $>99 \%$, Fluka) was performed on fully hydrogenated $\mathrm{Si}(111)$ : $\mathrm{H}$ surfaces using a UV-assisted $(\lambda=300 \mathrm{~nm}$ ) liquid phase process. In this study, no $\mathrm{SiO}_{2}$ component could be observed near $103 \mathrm{eV}$ on $\mathrm{Si} 2 \mathrm{p}$ photoelectron spectra taken at $\alpha=45^{\circ}$ immediately after grafting of the dodecyl molecular layer. The optical thickness $d_{\mathrm{SE}}=1.45 \mathrm{~nm}$ and the molecular coverage $\Sigma_{\mathrm{ML}}=2.3 \times 10^{14} \mathrm{~cm}^{-2}$ both indicate grafting of a rather dense $\mathrm{C}_{12}$ monolayer.

A low-doped $n$-type Si (1-10 $\Omega . c m$, Siltronix) has been chosen to obtain rectifying junctions. ${ }^{1,3,43-45}$ The doping density $\left(N_{\mathrm{D}}=1.5 \times 10^{14} \mathrm{~cm}^{-3}\right.$, as deduced from a Mott-Schottky plot of the high frequency capacitance, Fig. 6b) provides the position of the bulk Fermi level, $\left(E_{\mathrm{C}}-E_{\mathrm{FB}}\right)=q V_{\mathrm{N}}=k T \operatorname{Ln}\left(N_{\mathrm{C}} / N_{\mathrm{D}}\right)=0.306 \mathrm{eV}$ at $293 \mathrm{~K}$, where $N_{\mathrm{C}}\left(\mathrm{cm}^{-3}\right)=2.8 \times 10^{19}(T / 300)^{1 / 2}$ is the effective density of states in the Si conduction band.

For current density $J(V, T)$ and admittance $Y(V, T, \omega)$ measurements, a home-made parallel plate sample holder has been designed to insert a mercury drop on top of the molecular junction. ${ }^{15}$ This Teflon cell is inert towards mercury. A fresh mercury (99.999 \% Fluka) drop (contact area $S=5 \times 10^{-3} \mathrm{~cm}^{2}$ ) was used to avoid electrical shorts through pinholes in the OML. An ohmic back contact was made by applying a silver paste electrode on the scratched Si backside (inset of Fig. 4). Dc and ac transport measurements were performed in a cryostat under 
dry nitrogen flow to avoid water condensation and to minimize surface oxidation during the measurements.

$J(V)$ characteristics were measured in the dark, using a Keithley 6487 picoammeter. Admittance measurements were carried out with a frequency response analyzer (Alpha-A High Resolution measurement system, Novocontrol Technologies) using a two terminal active cell.. Experiments with $V_{\mathrm{AC}}=10 \mathrm{mV}$ were carried out in two runs, a voltage sweep (waiting time 0.3 second / step $25 \mathrm{mV})$ and a frequency sweep $\left(1 \times 10^{-2} \mathrm{~Hz}\right.$ to $\left.1 \times 10^{7} \mathrm{~Hz}\right)$, to study the measured complex admittance, $Y(V, T, \omega)=G_{\mathrm{m}}+j \omega C_{\mathrm{m}}$, against voltage, $V$, and frequency, $\omega / 2 \pi$.

The capacitance $(4.5 \mathrm{pF})$ of the empty Teflon cell in parallel with the molecular junction was subtracted to obtain $C_{m}$. Here, high frequency and high forward bias admittance data were not corrected for the series resistance $R_{S}$ (due to bulk Si and back contact resistance). $R_{S}$ effects were rather included in the modelling using the high frequency value of the real part of the impedance, $R_{S}=G_{\mathrm{m}} /\left(G_{\mathrm{m}}{ }^{2}+\omega^{2} C_{\mathrm{m}}{ }^{2}\right){ }^{22}$ The $R_{S}$ effect does not affect the excess capacitance $\hat{C}(0)-\hat{C}(\infty)$ or flat band voltage analysis; however, it broadens $M^{\prime \prime}(\omega)$ peaks and decreases the apparent response time.

\section{Data analysis}

A first criterion of the $\mathrm{Hg} / / \mathrm{C}_{12} \mathrm{H}_{25}-n$-Si junction quality is given by a cyclic $C(V)$ scan from $0 \mathrm{~V}$ to $-4 \mathrm{~V}$ (depletion) to $+0.8 \mathrm{~V}$ (accumulation near flat band conditions) to $0 \mathrm{~V}$, performed immediately after grafting, which does not show detectable hysteresis at any frequency (from $10^{2}$ $\mathrm{Hz}$ to $10^{6} \mathrm{~Hz}$ ) (Figure 6a).

\subsection{Current density}

Direct current characteristics $J(V, T)$ of the $\mathrm{Hg} / / \mathrm{C}_{12} \mathrm{H}_{25}-n$-Si junction show a strong rectification which increases with decreasing temperature (Fig. 4). At low forward bias $(0<V<$ 
0.4 Volt), the exponential $J(V)$ dependence, observed for all temperatures, is described by a thermionic emission (TE) mechanism. At high forward bias $(V>+0.6 \mathrm{~V})$, saturation in $J(V)$ and weak temperature dependence of $J(T)$ indicate that the current becomes limited by the tunnel barrier (TB) and by the contact resistance, rather than by the TE barrier inside the semiconductor. This behavior is typical of rectifying MIS tunnel junctions obtained previously with Hg // OML $n$ type $\mathrm{Si}^{1,3,43-45}$ In order to relate the applied voltage and the band bending, a full modeling of the current density, $J(V, T)$, characteristics has been reported elsewhere, ${ }^{15}$ including the applied voltage partition between the tunnel barrier, the space charge in the semiconductor and the voltage drop on the series resistance. Note that the tunnel barrier model (Eqn 3-5) incorporates the dc charge carrier transport mechanisms (tunneling, thermionic emission) which were analyzed previously. $^{15}$

\subsection{Admittance characteristics}

The frequency dependence of the admittance $Y(\omega)$ characteristics of the $\mathrm{Hg} / / \mathrm{C}_{12} \mathrm{H}_{25}-n$-Si junction is shown in Figures 5-7. In Figure 5, the zero bias conductance $G(\omega)$ shows three frequency regimes in the range $1 \times 10^{-2} \mathrm{~Hz}$ to $1 \times 10^{7} \mathrm{~Hz}$. Considering $G(\omega)$ at $243 \mathrm{~K}$, at low frequencies (typically below $1 \mathrm{~Hz}$ ), the conductance value $G(\omega \approx 0)$ is similar to the differential conductance $G=\mathrm{d} J / \mathrm{d} V$ derived from the dc $J(V)$ characteristics in Fig. 4; from reverse to forward bias, it increases with increasing forward bias over seven decades $\left(10^{-11}\right.$ to $10^{-4}{\mathrm{~S} . \mathrm{cm}^{-2}}$ at $243 \mathrm{~K}$, not shown). At intermediate frequencies (typically above $1 \mathrm{~Hz}$ for reverse applied bias), a power law dependence, $G(\omega) \approx \omega^{p}$, is observed; it holds with an exponent $p=0.91 \pm 0.03$ over nearly three decades in $G(T=243 \mathrm{~K}, V=0 \mathrm{~V})$. At high frequencies (typically above $1 \mathrm{MHz}$ ), the observed behaviour is dominated by the series resistance $R_{s}$ which gives a quadratic frequency dependence of the measured conductance $G_{m}(\omega)$ as $\omega^{2} C^{2} R_{s}$; such behaviour is expected from a $\mathrm{R} / / \mathrm{C}$ circuit in series with a resistor $R_{S}$ : 
$G_{m}(\omega)=\frac{G\left(1+G R_{S}\right)+\omega^{2} C^{2} R_{S}}{\left(1+G R_{S}\right)^{2}+\omega^{2} C^{2} R_{S}^{2}}$

if $\left(\omega_{S} \omega_{R C}\right)^{1 / 2} \ll \omega \ll \omega_{S}$ where $\omega_{S}=\left(C R_{S}\right)^{-1}$ and $\omega_{R C}=(G / C)$.

As shown in Figure 6, the capacitance at reverse bias $(V<+0.2 \mathrm{~V})$ is low, $C_{\mathrm{REV}}=10-20 \mathrm{pF}$ (2-4 nF.cm $\left.{ }^{-2}\right)$, at all frequencies, showing the formation of a depletion layer in the SC. It has been suggested that an inversion layer does not form because the hole tunnelling time is expected to be shorter than the generation-recombination time. ${ }^{46}$ In weak depletion $(+0.2 \mathrm{~V}<V<+0.7 \mathrm{~V}), C(V)$ data reveal a strong frequency dependence which indicates the additional contribution of interface states at lower frequencies; in the equivalent circuit (inset Fig. 6a), they correspond to a parallel $\left(R_{\mathrm{IT}} / / C_{\mathrm{IT}}\right)$ component. The measured low frequency capacitance at high forward bias is larger than the geometric capacitance of the molecular insulator, $C_{\mathrm{i}}=1.8 \mu \mathrm{F} . \mathrm{cm}^{-2}$, expected from $d_{\mathrm{T}}=$ $1.5 \mathrm{~nm}$ and $\varepsilon_{\tilde{i}}=3 .^{3}$ This result, $C(0)>C_{i}$, is specific of ultrathin MIS tunnel junctions with a low tunnel barrier resistance $R_{\mathrm{T}}$ (equivalent circuit, inset Fig. 6a); this relation is predicted by Eqn 14 if $q \beta J>C_{i}^{2} /\left(q^{2} D_{s} \tau\right)$, while the alternative condition, $D_{S}>C_{i} / q^{2}$ (corresponding to $1 \times 10^{13} \mathrm{eV}^{-1} \cdot \mathrm{cm}^{-2}$ ), is very unlikely for a device-quality junction.

In order to validate Equations 14-15 derived from the tunnel barrier model, Figure 7 shows the "excess capacitance" defined as $\left(C_{L F}-C_{H F}\right)=C(100 \mathrm{~Hz})-C(1 \mathrm{MHz})$. The low frequency response has been arbitrarily taken at $100 \mathrm{~Hz}$ but a lower frequency could be chosen; on this junction, the $10 \mathrm{~Hz}$ capacitance is basically similar to the $100 \mathrm{~Hz}$ data at $293 \mathrm{~K}$; however it shows a poorer signal / noise ratio (not shown). Note that this $C(100 \mathrm{~Hz})$ vs $J$ behaviour is weakly dependent on the measurement temperature $T$ in the range $253-293 \mathrm{~K}$, except for the low currents dominated by the thermionic emission mechanism (Figure 4).

(i) For reverse and small forward bias $\left(V<+0.25 \mathrm{~V}, J<7 \times 10^{-6} \mathrm{~A} . \mathrm{cm}^{-2}\right)$ corresponding to a Fermi level position below mid-gap, $C(100 \mathrm{~Hz})$ is very similar to the high frequency capacitance $C(1$ 
$\mathrm{MHz}$ ) (dashed line, $C_{S C}$, in Figure 7), because interface states below mid-gap are unable to respond to the ac voltage. Subtraction of the high frequency capacitance $C(1 \mathrm{MHz})$ from $C(100$ $\mathrm{Hz}$ ) leaves a very small low frequency contribution $\left(0.4 \mathrm{nF} . \mathrm{cm}^{-2}\right)$ independent of $J$ and $T$ (for $J<$ $2 \times 10^{-7}$ A.cm ${ }^{-2}$ ) ; in the depletion regime, this low frequency response adds to the semiconductor capacitance and thus explains the frequency shift in Mott-Schottky plots observed in Fig. $\mathbf{6 b}$.

(ii) For larger forward bias $\left(+0.25 \mathrm{~V}<V<+0.50 \mathrm{~V}, J<10^{-3}\right.$ A. $\left.\mathrm{cm}^{-2}\right)$ corresponding to a Fermi level position above mid-gap, a monotonic increase of the interface states response is essentially measured at $\omega \approx 100 \mathrm{~Hz}$; in this regime, the capacitance of the depletion layer $C(1 \mathrm{MHz})$ increases and remains smaller than $C(100 \mathrm{~Hz})$.

(iii) An abrupt transition occurs at $V=+0.50 \mathrm{~V}\left(J=10^{-3}\right.$ A. $\left.\mathrm{cm}^{-2}\right)$ with a steep increase in the slope; this inflexion point (blue arrow in Figure 7) marks the onset of the "high current density regime", where $\hat{C}(0)-\hat{C}(\infty) \approx q^{2} D_{s}\left(1+q \beta J \tau / C_{i}\right)$ becomes dominated by the product $J D_{s} \tau$. At the transition, $q \beta J \tau \approx C_{\mathrm{i}}$ provides the magnitude of the microscopic response time of interface defects, $\tau \approx 10 \mu$ s.

(iv) Above this transition, in the very high current density regime $\left(J>10^{-3} \mathrm{~A} \cdot \mathrm{cm}^{-2}\right)$ obtained for large forward bias $(+0.5 \mathrm{~V}<V<+0.80 \mathrm{~V})$, the dependence of the low frequency capacitance $C(\omega \approx 0)$ vs $J$ is superlinear, because both $J$ and $D_{S}$ increase simultaneously. Interestingly, a linear dependence of $C(100 \mathrm{~Hz})$ as a function of the current density is recovered in the very high current density regime, typically above $J=15 \mathrm{~mA} . \mathrm{cm}^{-2}$, close to the flat band voltage, $V_{\mathrm{FB}}=0.68$ V (Fig. 6b). The inset in Figure $7(293 \mathrm{~K})$ shows a linear dependence of $C(\omega \approx 0)$ vs $J$ as expected from Eqn 15 if $D_{s}$ is weakly dependent on the applied forward bias; this condition can be met if the Fermi level is pinned in a high density of interface states located near the Si conduction band. This hypothesis is reasonable if one considers the magnitude of $D_{\mathrm{S}} \approx 4 \times 10^{12}$ $\mathrm{eV}^{-1} \cdot \mathrm{cm}^{-2}$ derived from the experimental slope $\left(q^{3} D_{S} \beta \tau / C_{i}\right)$ and the microscopic response time of interface defects, $\tau \approx 10 \mu \mathrm{s}$. 


\subsection{Density of states distribution}

To obtain a reliable distribution of the interface defect density, $D_{S}(E)$, close to the conduction band, we investigate the forward bias range where the admittance of the thin MIS diode is modified by a high current density. In the following, $D_{S}(E)$ is derived using three complementary methods: (i) the low-high frequency capacitance (or excess capacitance) method, strictly valid for thick MIS diodes, ${ }^{22}$ (ii) the $f^{L F}$ (or response time) method; (iii) the $M_{M A X}^{L F}$ (or electrical modulus amplitude) method.

In the low-high frequency capacitance method, assuming $l=0$, the ac response of interface traps due to the modulation of their average occupation is described by a parallel capacitance resistance circuit in parallel with the space charge layer capacitance (inset of Fig. 6a). As shown in Fig. 6a, interface states do not respond at high frequencies, where the measured capacitance is essentially due to the SC; hence $D_{S}\left(E_{C}-E_{T}\right)$ can be obtained by subtracting the high frequency capacitance, $D_{S}=\left(C_{L F}-C_{H F}\right) / q S$; this approximation holds because $C_{\tilde{i}}$ is very large.

For the latter two methods, at each bias voltage, we use the measured electrical modulus (Fig. 8a) and the modulus calculated using the tunnel barrier model. The insulator capacitance is taken as $C_{\mathrm{i}}=1.8 \mu \mathrm{F} . \mathrm{cm}^{-2}$. An assumption has to be made on $V_{C}$ and $\tau$ values (Eqn. 7); using previous simulations, ${ }^{35} V_{C}$ was taken at the bias giving an ideality factor equal to 1.15 (here $q V_{C}=-3 \mathrm{kT}$ ) and the microscopic response time of interface defects, $\tau \approx 10 \mu \mathrm{s}$, was obtained at the transition (blue arrow in Figure 7).

In the $f^{L F}$ method (response time method reported previously ${ }^{15}$ ), admittance simulations use experimental $J(V)$ data (Fig. 4) and high frequency capacitance (1 MHz). $D_{S}$ values are obtained by matching the calculated device response time $\tau_{R}\left(V_{s} T_{s} D_{S}\right)$ values with experimental values of the device response time (open dots in Fig. 8b) given by the frequency $f^{L F}$ corresponding to the 
maximum in the imaginary part of the modulus ( $M$ ”) in Fig. 8a. In the electrical modulus amplitude method, we use the fact that $M_{M A X}^{L F}$ decreases with increasing $D_{S}$ (Fig. $\left.3 \mathbf{b}\right)$. Note that the current density was assumed to remain constant $\left(J=1 \times 10^{-7} \mathrm{~A} \cdot \mathrm{cm}^{-2}\right)$ in this set of simulations. The corresponding values have been reported in Fig. 8c (blue dots).

The role of a high $J$ through the MIS tunnel diode can be assessed by comparing the energy distributions of interface defects resulting from the different methods. The results shown in Fig. 8c globally confirm the low $D_{S}$ values $\left(D_{S}<10^{11} \mathrm{eV}^{-1} . \mathrm{cm}^{-2}\right)$ near mid-gap for the $\mathrm{C}_{12} \mathrm{H}_{25}-n \mathrm{Si}$ interface. They indicate that the exponential increase in $D_{S}\left(E_{C}-E_{T}\right)$ near the conduction band derived from the low-high frequency capacitance method is overestimated due to the large dc current density; however, the low-high frequency capacitance method appears to be reliable up to $1 \mathrm{~mA} \cdot \mathrm{cm}^{-2}$ (here $V \leq 0.45 \mathrm{~V}$ ). In contrast, the $M_{M A X}^{L F}$ method (within the low $J$ approximation) underestimates $D_{S}(E)$ as compared with the response time method.

\section{Discussion}

Before discussing the effect of the high current density, let us recall that several mechanisms may also affect the dynamic response of the MIS tunnel device, namely lateral inhomogeneity and dipolar relaxation effects. ${ }^{42}$

The power law dependence of the measured conductance, $G(\omega)$, is very different from the high frequency plateau expected for a MIS tunnel device with homogeneous barrier height. Similarly, some unexpected plateau appears over a broad frequency range in $M^{\prime \prime}(\omega)$ plots (Fig. 8a, reverse bias). As discussed in Section 2.4, this indicates that the junction has a distribution of low barrier height patches or thinner tunnel barriers. This conclusion is consistent with low temperature dc current characteristics of molecular tunnel junctions. ${ }^{42}$ Note that the excess capacitance will be less affected than the excess conductance since the space charge layer 
thickness is weakly dependent on the interface potential fluctuations. Hence, we expect that the homogeneous model still applies to experimental excess capacitance results.

For reverse and small forward bias $\left(V<+0.25 \mathrm{~V}, J<7 \times 10^{-6} \mathrm{~A} . \mathrm{cm}^{-2}\right)$ corresponding to a Fermi level position below midgap, interface states are unable to respond to the ac voltage. The observed very small low frequency capacitance being independent on temperature (in the investigated range $243-293 \mathrm{~K}$ ), it is rather attributed to some dipolar relaxation in the molecular monolayer, likely due to interface Si-C bond dipoles, in the absence of any detectable silicon oxide $\mathrm{SiO}_{\mathrm{x}}$ component in XPS spectra ( $\mathrm{Si} 2 \mathrm{p}$ core level, $\alpha=45^{\circ}$, not shown). This low frequency contribution (0.5 nF.cm $\left.{ }^{-2}\right)$, attributed to Si-C interface dipoles of the fresh $\mathrm{Hg} / / \mathrm{C}_{12} \mathrm{H}_{25}-\mathrm{Si}$ device, is much smaller than the dipolar capacitance values $\left(30-40 \mathrm{nF} . \mathrm{cm}^{-2}\right)$ reported in a recent admittance study of alkyl $\left(\mathrm{C}_{18}-n^{+} \mathrm{Si}\right)$ molecular assemblies. ${ }^{14}$

In MIS tunnel junctions with molecular insulator, low dipolar contribution is an important condition to detect a small surface states response, since the latter may be masked by any dipolar relaxation of interface or embedded polar moieties. As an illustration, in a study of long term stability of molecular devices (ageing at the ambient for eighteen months), a significant increase has been observed in the dipolar relaxation capacitance (x 50) and in the interface states response (x 7) of the $\mathrm{Hg} / / \mathrm{C}_{12} \mathrm{H}_{25}-\mathrm{Si}$ junction; besides the appearance of an interface silicon oxide layer revealed by XPS, some presence of adsorbed water vapour cannot be excluded. ${ }^{42}$ This effect could be possibly delayed or suppressed by engineering a very compact molecular layer. ${ }^{47}$

To understand the admittance response of interface states, we have proposed a representation of the excess capacitance, $\left(C_{L F}-C_{H F}\right)$, as a function of the dc current density, $I$, which clearly reveals the different regimes predicted by modelling the non equilibrium tunnel MIS junction in the forward bias range. Interestingly, the admittance behavior can be mapped onto the dc transport mechanisms ${ }^{15}$ : (i) in the low current density regime, the response of interface states above midgap is unaffected, corresponding to a current density limited by thermionic emission 
over the barrier in silicon; (ii) in the high current density regime $\left(J>1 \mathrm{~mA} \cdot \mathrm{cm}^{-2}\right)$, the admittance depends strongly on both the density of localized states and the dc current density, corresponding to a current density limited by tunneling through the molecular barrier.

At low current density values, a good agreement is found between the interface states distributions $D_{S}(E)$ derived from modeling of the device response frequency $f^{L F}$ and modulus amplitude $M_{M A X}^{L F}$ values, as compared with the low-high frequency capacitance method valid for thick MIS devices. Hence, for the $\mathrm{C}_{12} \mathrm{H}_{25}-n$ Si interface, the latter method appears to be reliable up to $1 \mathrm{~mA} . \mathrm{cm}^{-2}$. Future work is required to understand the discrepancies between $f^{L F}$ and $M_{M A X}^{L F}$ methods, which appear at intermediate current densities (Figure 8c).

The microscopic tunnel response time ( $\tau=10 \mu$ for the $\mathrm{C}_{12} \mathrm{H}_{25}-n$ Si interface) has been derived from the inflexion point in the excess capacitance, which marks the onset of the high current density regime (Eqn 15). This derivation requires an accurate estimate of the insulator capacitance per unit area; however, in ultra thin MIS diodes, $C_{\mathrm{i}}$ cannot be obtained from the measured capacitance saturation at high forward bias, in contrast with thick MIS.

In Figure 7, some increase in the $C(\omega \approx 0)$ values at high current density has been observed after the first voltage scan (up to $0.8 \mathrm{~V}$ ) at $293 \mathrm{~K}$. Subsequent scans at lower temperatures (273 K and $253 \mathrm{~K})$ show reproducible $C(\omega \approx 0)$ vs $/$ plots. Hence, more work is needed to understand the detailed shape of the $C(\omega \approx 0)-C(\omega \approx 1 \mathrm{MHz})$ vs $/$ plots at very high current densities, e.g. by performing ageing studies at the ambient or under high bias-stress conditions. In particular, it will be interesting to check if the linear part of the $C(\omega \approx 0)$ vs $/$ plot is observed in all molecular MIS diodes or if it is rather an accidental effect.

\section{Conclusion}


Modifications of the measured admittance induced by a high current density in ultrathin MIS devices have been investigated in order to obtain reliable values of the density distribution, $D_{S}(E)$, of defects localized at the semiconductor interface. The behavior of admittance and current density characteristics has been illustrated using $\mathrm{Hg} / / \mathrm{C}_{12} \mathrm{H}_{25}-\mathrm{Si}$ junctions incorporating 1-2 nm thick alkyl molecular layers covalently bonded to Si(111); using $n$-type silicon, the rectifying characteristics provide a very wide range of current densities.

Modeling the forward bias admittance of non equilibrium tunnel junctions reveals several regimes which can be observed either in $C(\omega \approx 0)$ vs $(J)$ plots of the low frequency capacitance over six decades in current or in $M^{w}(\omega)$ plots of the electrical modulus over eight decades in frequency. At low current density, the response of interface states above mid-gap is unaffected and a good agreement is found between the interface states densities derived from the modeling of either device response time $\tau_{\mathrm{R}}(V)$ and magnitude of the electrical modulus $M^{\prime \prime}(\omega)$, as compared with the low-high frequency capacitance method valid for thick MIS devices; the low value of $D_{S}(E)$ near mid-gap $\left(D_{S}<1 \times 10^{11} \mathrm{eV}^{-1} \cdot \mathrm{cm}^{-2}\right)$ indicates good passivation of dangling bonds at the $\mathrm{C}_{12} \mathrm{H}_{25}-n \mathrm{Si}$ interface.

In the high current density regime $\left(J>1 \mathrm{~mA} \cdot \mathrm{cm}^{-2}\right)$, the admittance depends strongly on both the density of localized states and the dc current density, so that the excess capacitance method overestimates $D_{s}$. For very high current densities $\left(J>10 \mathrm{~mA} \cdot \mathrm{cm}^{-2}\right)$, the linear $C(\omega \approx 0)$ vs $(J)$ dependence could indicate some Fermi level pinning in a high interface density of states near the Si conduction band.

The $C(\omega \approx 0)$ vs $(J)$ representation of dynamic characteristics of molecular layers covalently immobilized on $\mathrm{Si}(111)$ is also interesting to discriminate dipolar relaxation effects from interface states response. The temperature-independent excess capacitance $C(\omega \approx 0)-C(1 \mathrm{MHz})$ observed 
at very small current density, not predicted by the admittance model, is attributed to some dipolar relaxation in the molecular junction. Dipolar relaxation effects may obscure the interface states response for molecular insulator with strong interface or embedded dipolar moieties.

\section{Acknowledgments}

We wish to thank Jean-Paul Kleider (CNRS) for fruitful suggestions about transport mechanisms, Bruno Fabre and Cyril Herrier (CNRS - University of Rennes 1) for molecular grafting, Roopa Hiremath for some admittance measurements, Cristelle Mériadec for some XPS characterizations, Région Bretagne and Rennes Métropole for their financial support.

\section{REFERENCES}

[1] Salomon A, Boecking T, Seitz O, Markus T, Amy F, Chan CK, et al. What is the Barrier for Tunneling Through Alkyl Monolayers? Results from $n$ - and $p$-Si-Alkyl/Hg Junctions. Adv Mater 2007;19:445-50.

[2] Har-Lavan R, Ron I, Thieblemont F, Cahen D. Toward metal-organic insulator-semiconductor solar cells, based on molecular monolayer self-assembly on $n$-Si. Appl Phys Lett 2009;94:043308.

[3] Faber EJ, de Smet LCPM, Olthuis W, Zuilhof H, Sudholter EJR, Bergveld P, et al. Si-C Linked Organic Monolayers on Crystalline Silicon Surfaces as Alternative Gate insulators. ChemPhysChem 2005;6:2153-66.

[4] Aswal DK, Lenfant S, Guerin D, Yakhimi JV, Vuillaume D. Self assembled monolayers on silicon for molecular electronics. Anal Chimica Acta 2006;568:84-108.

[5] Lindsay SM, Ratner MA. Molecular Transport Junctions: Clearing Mists. Adv Mater 2007;19:23-31.

[6] Vuillaume D. New concepts for nanophotonics and nano-electronics. Molecular-scale electronics. CR Physique 2008;9:78-94.

[7] Vilan A, Yaffe O, Biller A, Salomon A, Kahn A, Cahen D. Molecules on Si: Electronics with chemistry. Adv Mater 2010;22:140-59.

[8] Ghosh AW, Electronics with molecules, Comprehensive Semiconductor Science and Technology (Elsevier, Amsterdam, 2011), Chap. 5.09, pp. 383-479. 
[9] Linford MR, Chidsey CED. Alkyl monolayers covalently bonded to silicon surfaces. J Am Chem Soc 1993;115:12631-2.

[10] Royea WJ, Juang A, Lewis NS. Preparation of air stable, low recombination velocity Si(111) surfaces through alkyl termination. Appl Phys Lett 2000;77:1988-90.

[11] Lehner A, Kohl F, Franzke SA, Graf T, Brandt MS, Stutzmann M. Photoconductivity and spin dependent photoconductivity of hydrosylilated (111) silicon surfaces. Appl Phys Lett 2003;82:565-7.

[12] Kar S, Miramond C, Vuillaume D. Properties of electronic traps at silicon/1-octadecene interfaces. Appl Phys Lett 2001;78:1288-90.

[13] Kar S. Study of silicon-organic interfaces by admittance spectroscopy. Appl Surf Sci 2006;252:3961-7.

[14] Clement N, Pleutin S, Guerin D, Vuillaume D. Relaxation dynamics in covalently bonded organic monolayers on silicon. Phys Rev B 2010;82:035404.

[15] Fadjie-Djomkam AB, Ababou-Girard S, Hiremath R, Herrier C, Fabre B, Solal F, Godet C. Temperature dependence of current density and admittance in metal-insulator-semiconductor junctions with molecular insulator. J Appl Phys 2011;110:083708.

[16] Thuo MM, Reus WF, Nijhuis CA, Barber JR, Kim C, Schulz MD, Whitesides GM. Odd-even effects in charge transport across self-assembled monolayers. J Am Chem Soc 2011;133:2962-75.

[17] Smaali K, Clement N, Patriarche G, Vuillaume D. Conductance statistics from a large array of sub10 nm molecular junctions. ACS Nano 2012;6: 4639-4647.

[18] Sze SM. Semiconductor devices: Physics and Technology, Wiley, New York, 1985.

[19] Card HC, Rhoderick EH. Studies of tunnel MOS diodes I. Interface effects in silicon Schottky diodes. J Phys D: Appl Phys 1971;4:1589-601.

[20] Wu CY. Interfacial layer theory of the Schottky barrier diodes. J Appl Phys 1980;51:3786-89.

[21] Wu CY. Interfacial layer-thermionic-diffusion theory for the Schottky barrier diode. J Appl Phys 1982;53:5947-50.

[22] Nicollian EH, Brews JR. MOS (Metal Oxide Semiconductor) Physics and Technology, Wiley, New York, 1982.

[23] Castagne R, Vapaille A. Description of the $\mathrm{SiO}_{2}$-Si interface properties by means of very low frequency MOS capacitance measurements. Surf Sci 1971;28:157-193.

[24] Tseng HH, Wu CY. A simple interfacial model for the non-ideal I-V and C-V characteristics of the Schottky-barrier diode. Solid-State Electron 1987;30:383-90.

[25] Werner J, Ploog K, Queisser HJ. Interface states measurements at Schottky contacts: a new admittance technique. Phys Rev Lett 1986;57:1080-4. 
[26] Kar S, Dahlke WE. Interface states in MOS structures with 20-40 $\AA$ thick $\mathrm{SiO}_{2}$ films on nondegenerate Si. Solid-State Electron 1972;15:221-37.

[27] Viktorovitch P. Bulk and surface states analysis in a-Si:H by Schottky and MIS tunnel diodes capacitance and conductance measurements. J Appl Phys 1981;52:1392-404.

[28] Ouennoughi Z, Sella A. MIS tunnel admittance with an inhomogeneous dielectric. Int J Electron 1997;83:571-80.

[29] Chattopadhyay P. Admittance of metal-insulator-semiconductor tunnel contacts in the presence of donor-acceptor mixed interface states and interface reaction. J Appl Phys 2001;89:364-373.

[30] Liu YJ, Yu HZ. Molecular passivation of mercury-silicon (p-type) diode junctions: alkylation, oxidation, and alkylsilation. J Phys Chem B 2003;107:7803-11.

[31] Pleutin S, Clement N, Guerin D, Vuillaume D. Molecular relaxation dynamics in organic monolayer junctions. Phys Rev B 2010;82:125436.

[32] Scott MC, Stevens DR, Bochinski JR, Clarke LI. Dynamics within alkylsiloxane self-assembled monolayers studied by sensitive dielectric spectroscopy. ACS Nano 2008;2: 2392-2400.

[33] Zhang Q, Zhang Q, Archer LA. Molecular relaxation dynamics of self-assembled monolayers. J Phys Chem B 2006;110:4924.

[34] Sune J, Oriols X, Autran JL. Non-equilibrium gate tunneling current in ultra-thin $(<2 \mathrm{~nm})$ oxide MOS devices. J Non-Cryst Solids 2001;280:127-131.

[35] Gomila G, Rubi JM. Relation for the nonequilibrium population of the interface states: Effects on the bias dependence of the ideality factor. J Appl Phys 1997;81:2674-81.

[36] Gomila G. Effects of interface states on the non-stationary transport properties of Schottky contacts and metal-insulator-semiconductor tunnel diodes. J Phys D: Appl Phys 1999;32:64-71.

[37] Werner JH. Electrical characterization of interface states at Schottky contacts and MIS tunnel diodes. In Metallization and Metal-semiconductor interface states (Plenum, New York, 1989), pp. 235-56.

[38] Chattopadhyay P, Raychaudhury B. Frequency dependence of forward capacitance-voltage characteristics of Schottky barrier diodes. Solid-State Electron 1993;36:605-610.

[39] Werner JH, Güttler HH. Barrier inhomogeneities at Schottky contacts. J Appl Phys 1991;69:15221533.

[40] Sullivan JP, Tung RT, Pinto MR, Graham WR. Electron transport of inhomogeneous Schottky barriers : A numerical study. J Appl Phys 1991;70:7403-7424.

[41] Tung RT. Recent advances in Schottky barrier concepts. Mat Sci Eng R 2001;35:1-138. 
[42] Fadjie-Djomkam AB, Ababou-Girard S, Godet C. Barrier height distribution and dipolar relaxation in metal-insulator-semiconductor junctions with molecular insulator: ageing effects. J Appl Phys 2012. [http://dx.doi.org/10.1063/1.4767121]

[43] Salomon A, Boecking T, Chan CK, Amy F, Girshevitz O, Cahen D, Kahn A. How Do Electronic Carriers Cross Si-Bound Alkyl Monolayers? Phys Rev Lett 2005;95:266807.

[44] Maldonado S, Plass KE, Knapp D, Lewis NS. Electrical Properties of Junctions between Hg and Si(111) Surfaces Functionalized with Short-Chain Alkyls. J Phys Chem C 2007;111:17690-9.

[45] Godet C, Fadjie A, Ababou-Girard S, Solal F. Tunnel barrier parameters derivation from normalized differential conductance in Hg / organic monomolecular layer-Si junctions. Appl Phys Lett 2010;97:132105.

[46] Kar S. Determination of minority carrier lifetime using MIS tunnel diodes. Appl Phys Lett 1974;25:587-9.

[47] Clement N, Guerin D, Pleutin S, Godey S, Vuillaume D. Role of hydration on the electrical transport through molecular junctions on silicon. J Phys Chem C 2012;116:17753-63. 


\section{FIGURE CAPTIONS}

Figure 1 Energy band diagram and carrier exchange mechanisms at the tunnel MIS interface ${ }^{36}$ (reprinted with permission from IOP).

Figure 2 Simulation of capacitance $C(\omega)$, conductance $G(\omega)$ and imaginary electrical modulus $M$ ” $(\omega)$ of the MIS tunnel junction using measured $J(V)$ (Fig. 4, $293 \mathrm{~K}$ ) and capacitance at 1 MHz. Continuous lines: at $+0.2 \mathrm{~V}$ forward bias for variable density of interface states, $D_{\mathrm{S}}$, in the range $10^{10}-3 \times 10^{12} \mathrm{eV}^{-1} \cdot \mathrm{cm}^{-2}$. Symbols: at fixed $D_{\mathrm{S}}=3 \times 10^{12} \mathrm{eV}^{-1} \cdot \mathrm{cm}^{-2}$ for variable forward bias.

Figure 3 a) Simulation of $M^{\prime \prime}(\omega)$ peaks (using $J=1 \times 10^{-7} \mathrm{~A} \cdot \mathrm{cm}^{-2}, C_{i}=1.8 \mu \mathrm{F} \cdot \mathrm{cm}^{-2}, C_{S C}=3.6$ $\left.\mathrm{nF} . \mathrm{cm}^{-2}\right)$; b) dependence of characteristic frequencies, $f^{L F}$ and $f^{H F}$, and magnitude of the modulus peak values, $M_{M A Z}^{L F}$ and $M_{M A R}^{H F}$, as a function of $D_{S}$.

Figure 4 Temperature dependence of current density $J(V)$ characteristics of a $\mathrm{Hg} / / \mathrm{C}_{12} \mathrm{H}_{25}-n-\mathrm{Si}$ junction in the range 243-293 K (step $10 \mathrm{~K}$ ). Inset: schematic of the MIS structure (note that the molecular layer thickness is not scaled) inserted within the Teflon cell.

Figure 5 Frequency dependence of the measured conductance of a $\mathrm{Hg} / / \mathrm{C}_{12} \mathrm{H}_{25}-n$-Si junction at zero bias and variable temperature $T$ ( $243 \mathrm{~K}$ to $293 \mathrm{~K}$ ) ; in the intermediate frequency range, a power-law behaviour is observed ( $p=0.91$ at $253 \mathrm{~K}$ ) with a trend towards saturation above $10^{5}$ Hz sketched by the dotted line. The series resistance, $R_{S}$ can be obtained from the high frequency regime where $G(\omega)$ is proportional to $\omega^{2}$. 
Figure 6 Frequency dependence of the measured capacitance of a $\mathrm{Hg} / / \mathrm{C}_{12} \mathrm{H}_{25}-n$-Si junction at $T=253 \mathrm{~K}:$ a) $C_{\mathrm{m}}(V)$ from $100 \mathrm{~Hz}$ to $1 \mathrm{MHz}$ showing the absence of hysteresis, b) MottSchottky plot ( $C_{\mathrm{m}}^{-2}$ vs $V$ ) giving the flat band voltage; the shift in the reverse bias range is attributed to a small dipolar relaxation component.

Figure 7 Excess capacitance $\left(C_{L F}-C_{H F}\right)$ as a function of the dc current density in a $\mathrm{Hg} / / \mathrm{C}_{12} \mathrm{H}_{25}$ - $n$-Si junction at $T=293 \mathrm{~K}$ (red crosses), $273 \mathrm{~K}$ (blue dots) and $253 \mathrm{~K}$ (green triangles). The voltage scale and the inset correspond to $293 \mathrm{~K}$ data. The inflection point (vertical arrow) corresponds to the transition between the low (TE limited) and the high (tunnel limited) current density regimes. The horizontal baseline $\left(2 \mathrm{pF}\right.$ or $\left.0.4 \mathrm{nF} . \mathrm{cm}^{-2}\right)$ is attributed to a small dipolar relaxation component.

Figure 8 : a) Experimental $M$ ” $(\omega)$ of a $\mathrm{Hg} / / \mathrm{C}_{12} \mathrm{H}_{25}-n$-Si junction measured at $293 \mathrm{~K}$ for increasing bias applied on $\mathrm{Hg}(-0.8 \mathrm{~V},-0.5 \mathrm{~V},-0.2 \mathrm{~V},-0.05 \mathrm{~V}, 0 \mathrm{~V},+0.05 \mathrm{~V},+0.1 \mathrm{~V},+0.2 \mathrm{~V}$, $+0.3 \mathrm{~V},+0.4 \mathrm{~V},+0.5 \mathrm{~V},+0.6 \mathrm{~V},+0.7 \mathrm{~V},+0.8 \mathrm{~V}$ ); b) experimental response time (circles) compared with simulated values (lines) using variable density of interface states $D_{S}$ from $1 \times 10^{10}$ to $3 \times 10^{12} \mathrm{eV}^{-1} \cdot \mathrm{cm}^{-2}$ (measured $J(V)$ were used as input in the simulation) ; c) energy dependence of the density of interface states $D_{S}\left(E_{C}-E_{T}\right)$ derived from experimental data using the low-high frequency capacitance method (black triangles), the $f^{L F}$ method (open squares), and the $M_{M A X}^{L F}$ method (full circles) as described in Section 4.3. The Fermi level is at mid gap for a forward applied bias of $+0.25 \mathrm{~V}$. 


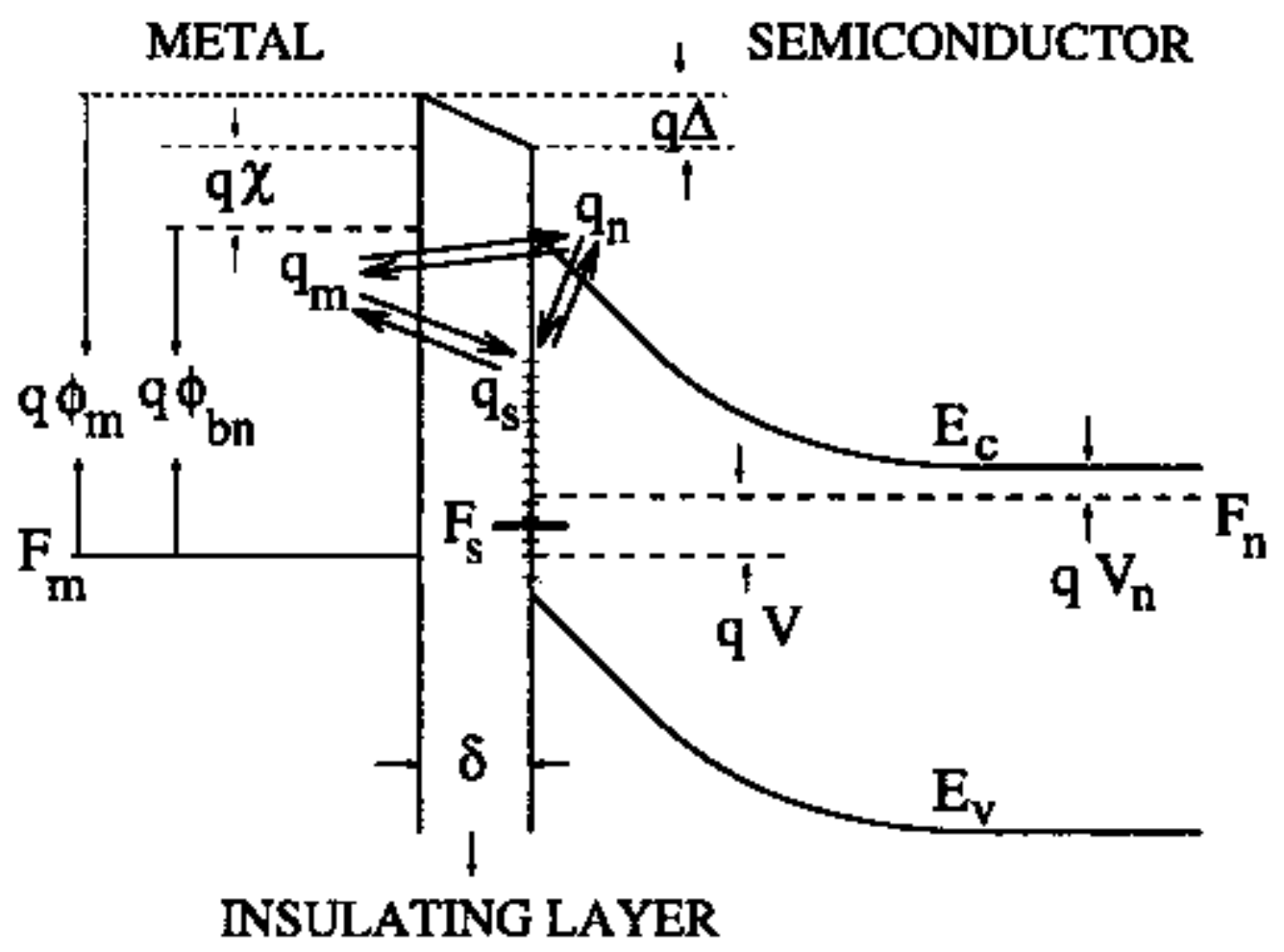

Figure 1 

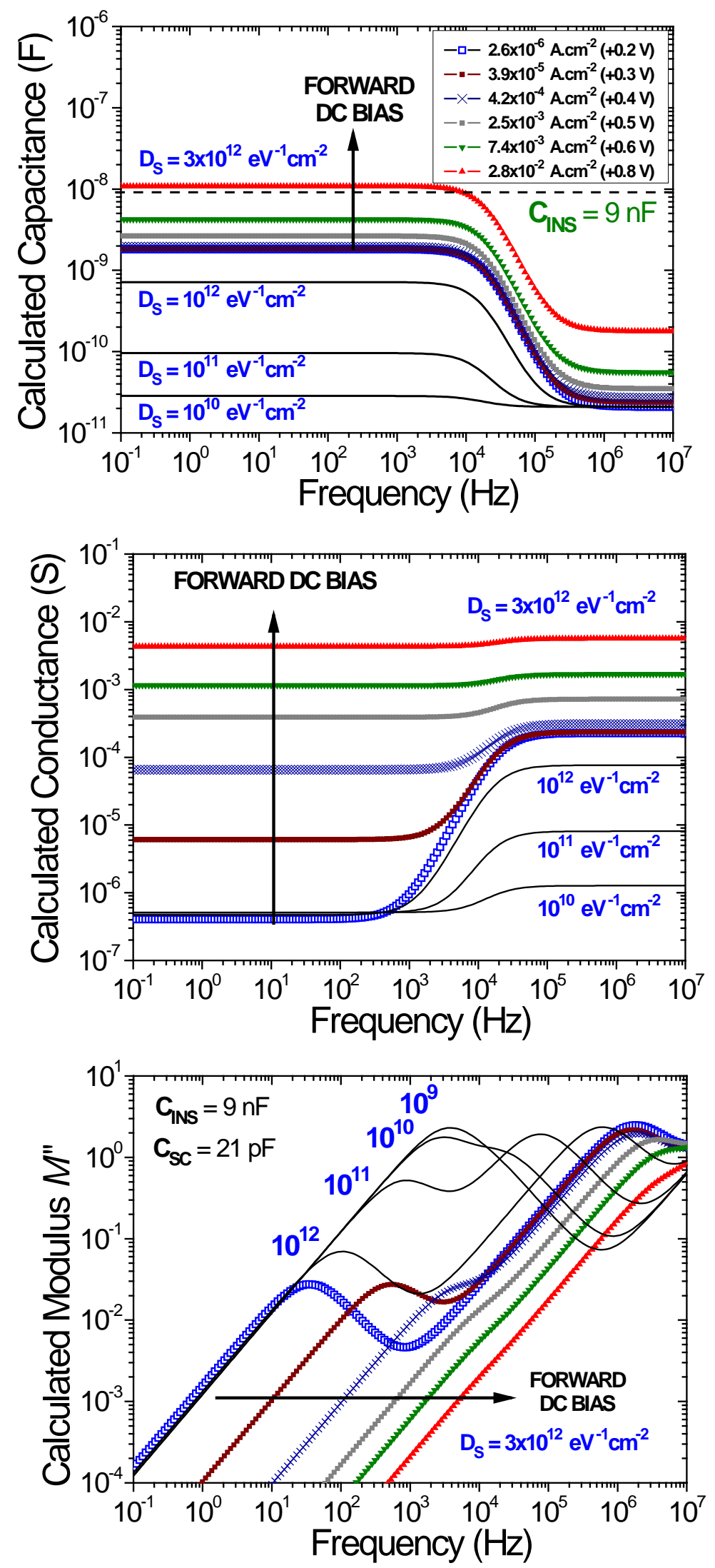

Figure 2 

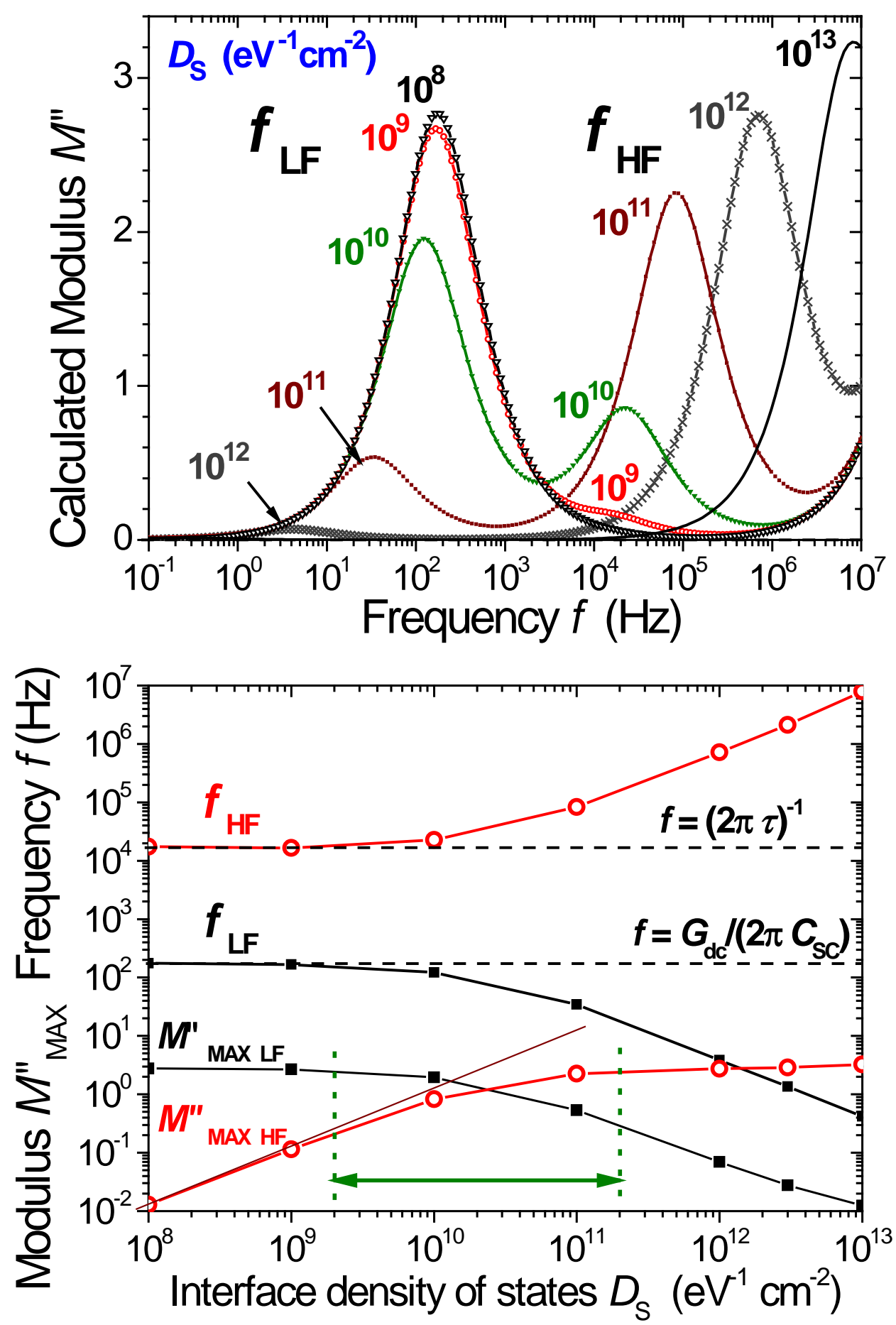

Figure 3 


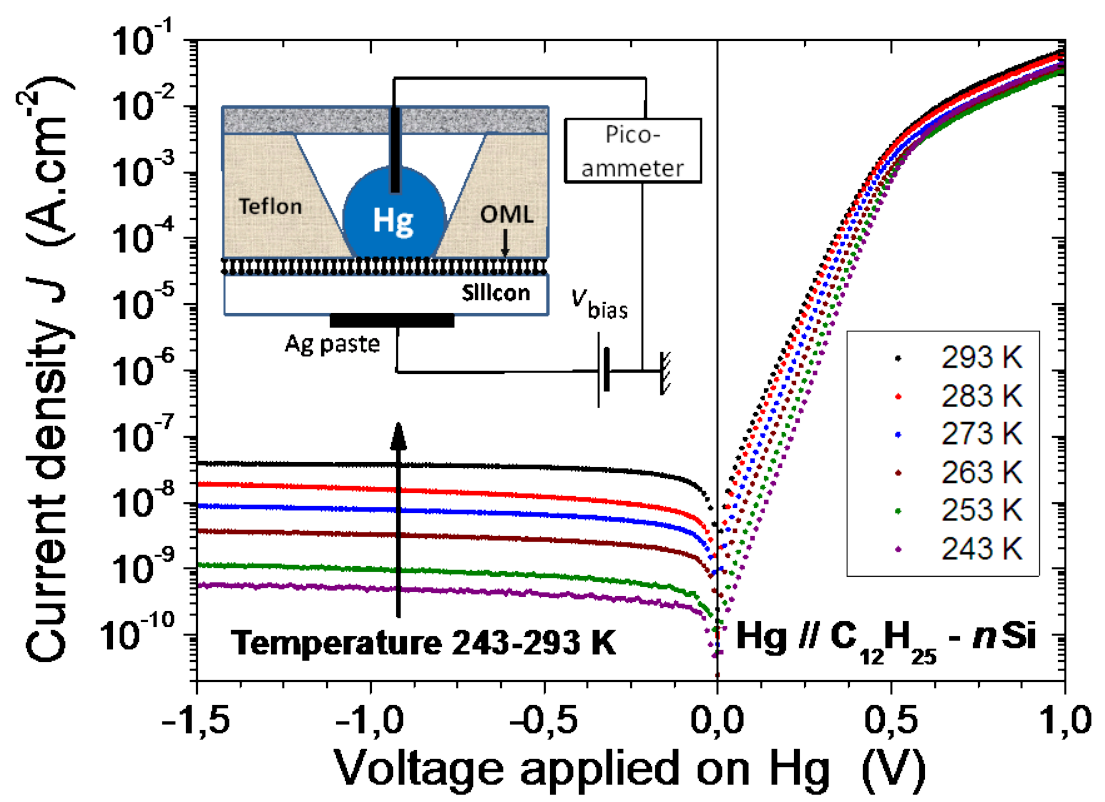

Figure 4 


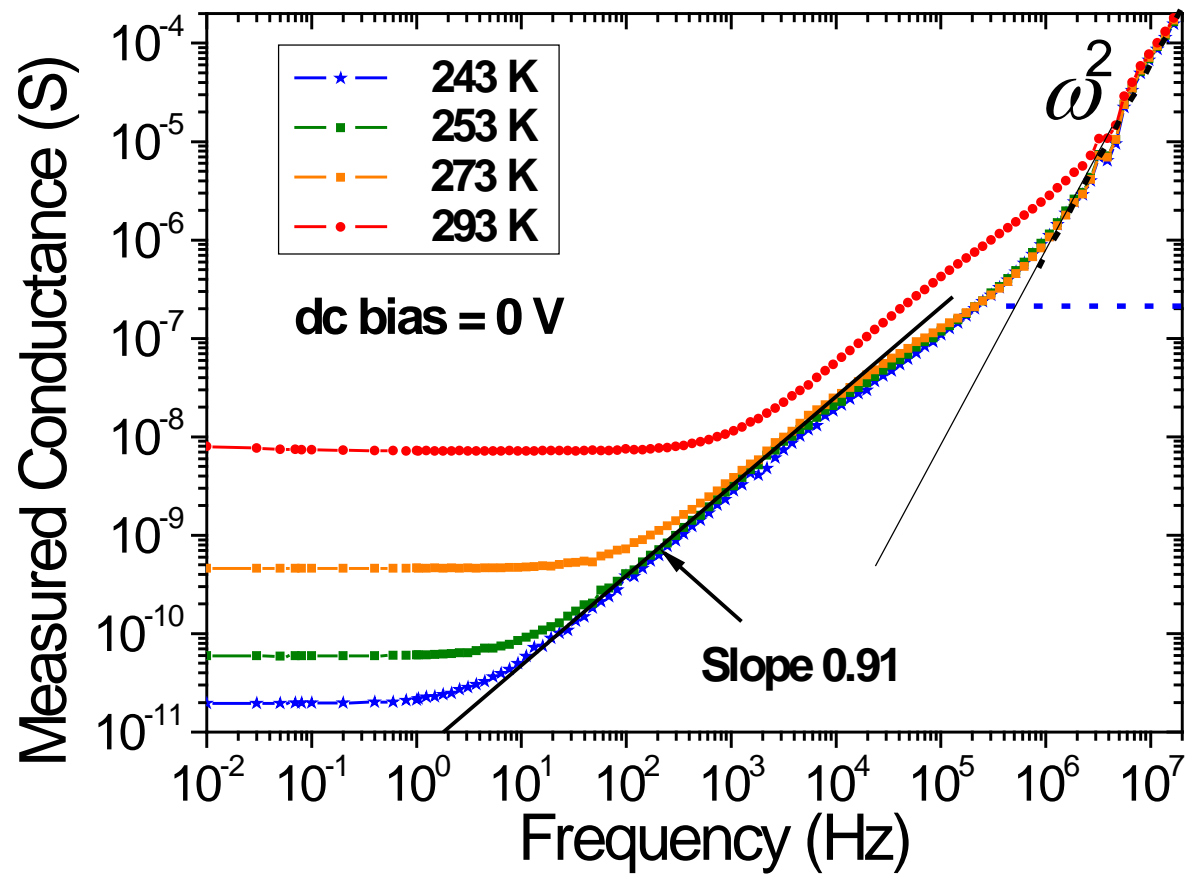

Figure 5 

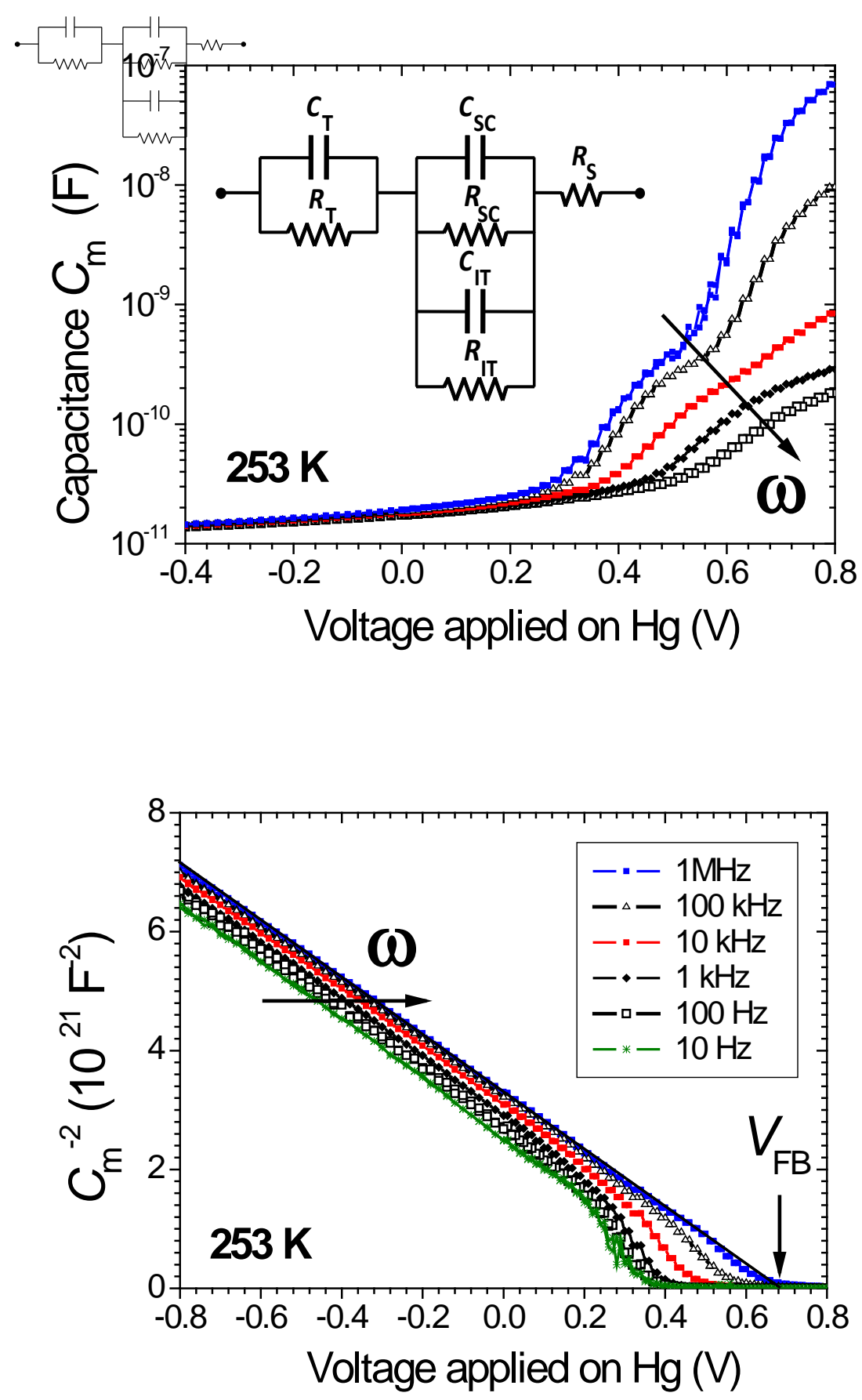

Figure 6 


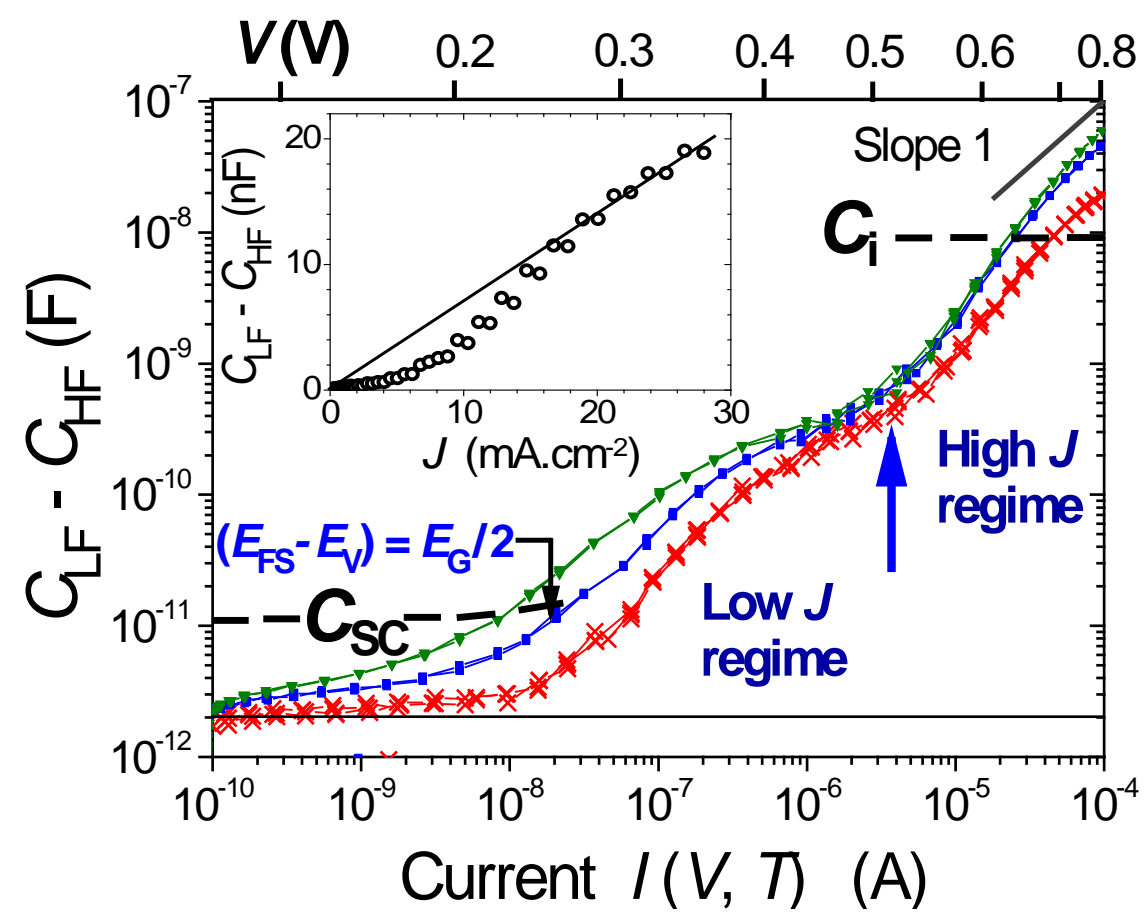

Figure 7 

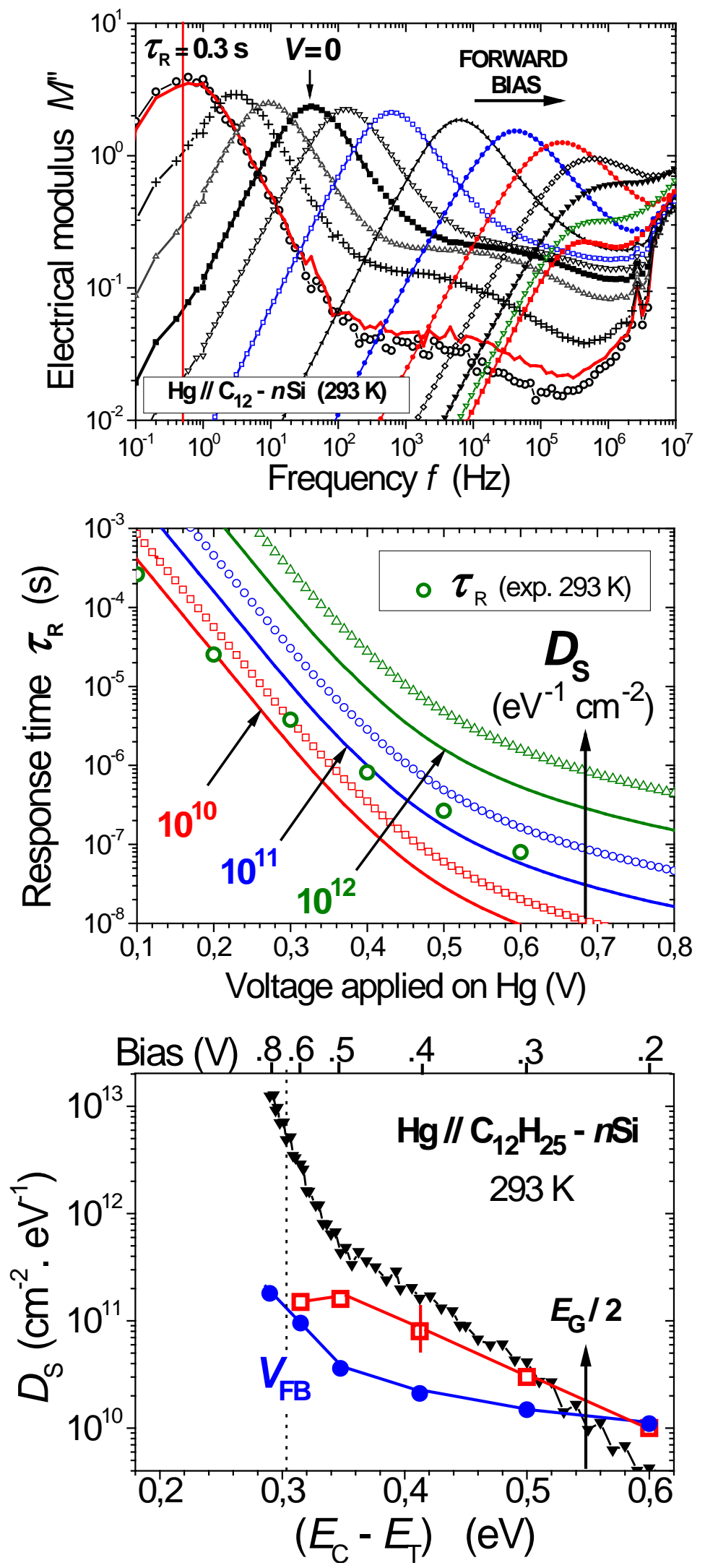

Figure 8 